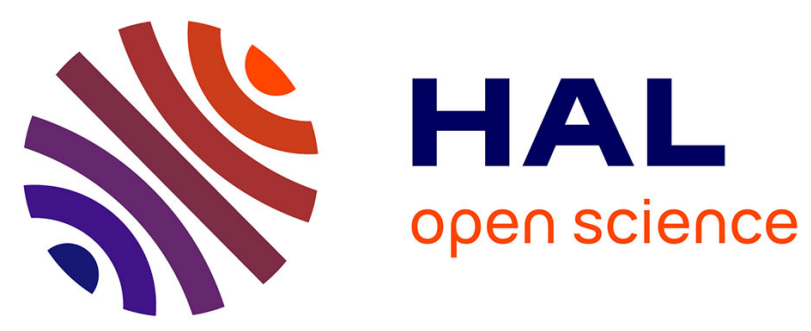

\title{
Salt-Responsive Pickering Emulsions Stabilized by Functionalized Cellulose Nanofibrils
}

James C Courtenay, Yun Jin, Julien Schmitt, Kazi M Zakir Hossain, Najet Mahmoudi, Karen J Edler, Janet L Scott

\section{- To cite this version:}

James C Courtenay, Yun Jin, Julien Schmitt, Kazi M Zakir Hossain, Najet Mahmoudi, et al.. SaltResponsive Pickering Emulsions Stabilized by Functionalized Cellulose Nanofibrils. Langmuir, 2021, 37, pp.6864 - 6873. 10.1021/acs.langmuir.0c03306 . hal-03443386

\section{HAL Id: hal-03443386 https://hal.science/hal-03443386}

Submitted on 23 Nov 2021

HAL is a multi-disciplinary open access archive for the deposit and dissemination of scientific research documents, whether they are published or not. The documents may come from teaching and research institutions in France or abroad, or from public or private research centers.
L'archive ouverte pluridisciplinaire HAL, est destinée au dépôt et à la diffusion de documents scientifiques de niveau recherche, publiés ou non, émanant des établissements d'enseignement et de recherche français ou étrangers, des laboratoires publics ou privés. 


\title{
Salt-Responsive Pickering Emulsions Stabilized by Functionalized Cellulose Nanofibrils
}

\author{
James C. Courtenay, ${ }^{\#}$ Yun Jin, ${ }^{\#}$ Julien Schmitt, ${ }^{\#}$ Kazi M. Zakir Hossain, Najet Mahmoudi,
} Karen J. Edler,* and Janet L. Scott*

Cite This: Langmuir 2021, 37, 6864-6873

Read Online

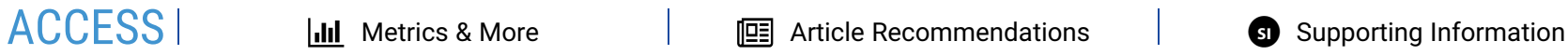

ABSTRACT: Oil-in-water emulsions have been stabilized by functionalized cellulose nanofibrils bearing either a negative (oxidized cellulose nanofibrils, OCNF) or a positive (cationic cellulose nanofibrils, CCNF) surface charge. The size of the droplets was measured by laser diffraction, while the structure of the shell of the Pickering emulsion droplets was probed using small-angle neutron scattering (SANS), confocal laser scanning microscopy (CLSM), scanning electron microscopy (SEM), and rheology measurements. Both OCNF- and CCNF-stabilized emulsions present a very thick shell $(>100 \mathrm{~nm})$ comprised of densely packed CNF. OCNF-stabilized

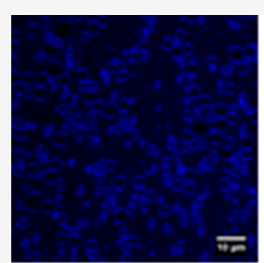

OM

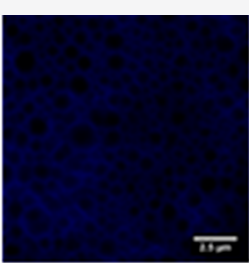

$0.1 \mathrm{M}$

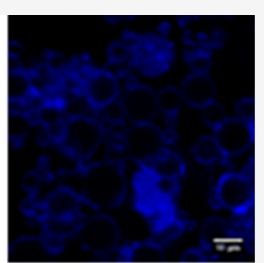

$0.5 \mathrm{M}$

$[\mathrm{NaCl}]$ emulsions proved to be salt responsive, influencing the droplet aggregation and ultimately the gel properties of the emulsions, while CCNF emulsions, on the other hand, showed very little saltdependent behavior.

\section{INTRODUCTION}

Interest in biobased materials has grown significantly in recent years in response to a growing awareness of the need for chemicals and materials based on renewable resources rather than fossil carbon (e.g., petroleum). Furthermore, the design requirement for materials with "appropriate lifetimes", 1 such that they do not persist in the environment once discarded, has focused attention on biobased and biodegradable materials such as cellulose. It is estimated that $1.5 \times 10^{12}$ tons of cellulose waste are produced annually from the wood pulp industry alone, so this is both a renewable and a cost-effective raw material source. ${ }^{2}$ The latter is important if materials are to move from being scientific curiosities to ingredients in large-scale, cost-sensitive applications, such as consumer products. Cellulose in its native form, however, is not easily water dispersible, limiting its direct application in water-based formulations. Cellulose nanocrystals (CNC) can be formed via sulfuric acid hydrolysis as needle-like particles dispersible in water. ${ }^{3}$ A cheaper alternative, the TEMPO $/ \mathrm{NaBr} / \mathrm{NaOCl}$ oxidation of cellulose, results in larger particles called oxidized cellulose nanofibrils (OCNF)., OCNF have been previously used as rheology modifiers with potential in low surfactant content personal care products. ${ }^{6,7}$ OCNF, along with $\mathrm{CNC}$, can be used as particulate emulsion stabilizers, in high-alcohol formulations, ${ }^{8}$ and in Pickering emulsions, which find application in surfactant-free cream formulations. ${ }^{9}$

Pickering emulsions are stabilized by solid particles adsorbed at the interface between two immiscible phases ${ }^{10}$ and are kinetically stable due to the steric hindrance and/or electrostatic repulsion between solid particles acting as barriers between droplets, thus preventing coalescence. ${ }^{11}$ By careful choice of the Pickering agents, Pickering emulsions can be tailored to ge $^{12}$ by modifying the $\mathrm{pH},{ }^{13,14}$ ionic strength, ${ }^{13,14}$ or temperature ${ }^{15,16}$ of the system. The key parameter to control the stability of Pickering emulsions is the wettability of the Pickering agent. ${ }^{17}$ Particles at the oil/water interface that have a contact angle $\theta \leq$ $90^{\circ}$ have a more hydrophilic behavior and hence tend to stabilize oil-in-water (o/w) emulsions, while water-in-oil (w/o) emulsions tend to form if more hydrophobic particles are used with a contact angle $\geq 90^{\circ} .{ }^{18,19}$ Spherical charged particles often require the addition of salt or surfactant to overcome the repulsion barrier preventing a dense enough packing at the interface of the emulsion droplets. ${ }^{20}$ In contrast, particles with a large aspect ratio such as rod-like particles or ellipsoids have been shown to exhibit strong attractive capillary forces at the oil/ water interface, allowing the formation of stable emulsions even when particles experience electrostatic repulsion. Oil-in-water emulsions stabilized using cellulose nanocrystals, ${ }^{14,21-24}$ nanofibrils, $^{25-27}$ or microcrystals ${ }^{28,29}$ have been previously investigated.

Received: November 17, 2020

Revised: April 29, 2021

Published: June 3, 2021 
Cellulose nanocrystals (CNC) have been applied as Pickering particles, ${ }^{14,21-24}$ both "as isolated" after sulfuric acid hydrolysis (thus bearing surface sulfate ester groups ${ }^{30-32}$ ) and desulfated using hydrochloric acid to decrease the electrostatic repulsion between CNC. ${ }^{33}$ Addition of low amounts of salt to CNCstabilized emulsions proved to enhance the stability of the droplets, allowing the formation of stable emulsions at a lower concentration of CNC. ${ }^{14,23}$ An extra TEMPO $/ \mathrm{NaBr} / \mathrm{NaOCl}$ oxidation step to introduce additional surface carboxylate groups to the CNC surface and improve the dispersion of the particles proved to prevent sufficient coverage of the droplets and hence stabilization of the emulsion. ${ }^{34}$ On the other hand, modification of the oxidized CNC surfaces by adsorption of quaternary ammonium salts bearing long alkyl "tails" yielded hydrophobic particles that stabilized w/o emulsions. ${ }^{34}$ Capron and co-workers previously provided a structural description of the interface of $\mathrm{CNC}$-stabilized o/w emulsions. ${ }^{33} \mathrm{CNC}$ were found to form monolayers on the surface of oil droplets with variable coverage and little or no penetration into the oil phase. It was suggested that the $\left(\begin{array}{lll}2 & 0 & 0\end{array}\right)$ crystal plane interacted directly with the interface. Finally, by grafting poly $(\mathrm{N}$-isopropylacrylamide) (PNIPAM), a thermoresponsive polymer, to the surface of $\mathrm{CNC}$, it is possible to form an emulsion that will break when submitted to temperatures above the lower critical solution temperature of the PNIPAM $\left(32^{\circ} \mathrm{C}\right) .{ }^{35}$ Similarly, $\mathrm{pH}$ sensitivity can be obtained by grafting poly[2-(dimethylamino)ethyl methacrylate . $^{36}$

Moreover, uncharged cellulose nanofibrils (CNF) obtained from mechanical disintegration were also added to surfactantstabilized oil/water emulsions to form w/o/w emulsions, enhancing the stability of the system, notably by increasing its viscosity. ${ }^{37}$ Alternatively, CNF were also added to CNCstabilized emulsions, successfully preventing creaming thanks to depletion stabilization. ${ }^{38}$ Emulsions were subsequently prepared using either uncharged or TEMPO-oxidized cellulose nanofibrils. ${ }^{27}$ Both were found to stabilize emulsions, not only via particle adsorption at the droplet surface but also by the presence of a cellulose nanofibril hydrogel network in the water phase. Moreover, it was shown that the TEMPO-oxidized nanofibrils improved the stability of the emulsion upon aging as the carboxyl groups induce repulsive forces between the droplets thanks to osmotic pressure. ${ }^{27,39}$ However, recent work from Syverud and co-workers showed a loss of the stabilization of emulsions with OCNF in the presence of salt at a very low concentration of nanofibrils. ${ }^{26}$ Recently, Isogai and co-workers also studied the use of OCNF as Pickering agents for the formation of emulsions. ${ }^{25}$ They showed that the higher the oil/ water interfacial tension, the higher the amount of nanofibrils required to stabilize the emulsions. Finally, Jiménez-Saelices and Capron compared oil-in-water emulsions stabilized by CNC, $\mathrm{CNF}$, or OCNF in the presence of $50 \mathrm{mM} \mathrm{NaCl}{ }^{40}$ They showed notably that emulsions stabilized by CNC were well dispersed in the water phase, while those stabilized by OCNF formed aggregates of droplets. Moreover, the size of the droplets could be decreased to form nanoemulsions via the use of high-pressure homogenization.

Here, we compare oil-in-water Pickering emulsions formed by cellulose nanofibrils bearing either a negative or a positive surface charge. Contrary to $\mathrm{CNC}$, the selected nanofibrils evidence crystalline regions linked by amorphous defects. ${ }^{41}$ They hence often present larger aspect ratios and high surface charges and thus provide excellent viscosity modification in concentrated supensions. ${ }^{42,43}$ In addition, the methodology used to prepare OCNF does not require strong acid hydrolysis, and thus, the mass yield of the material is $4-5$ times greater than that of CNC, making such materials more attractive for applications requiring industrial-scale production. The negatively charged nanofibrils were obtained from TEMPO oxidation (or by electrochemical oxidation ${ }^{44}$ ) and the positively charged nanofibrils (cationic cellulose nanofibrils or CCNF) by reaction of glycidyl trimethylammonium chloride (GTMAC) with surface $\mathrm{OH}$ groups in the presence of $\mathrm{NaOH} .{ }^{43,45}$ Both surface derivatization methods allow precise control of the degree of modification of the nanofibril surface. ${ }^{5,45}$

We studied the OCNF- or CCNF-stabilized emulsions using laser diffraction for droplet size analysis, microscopy techniques, and small-angle neutron scattering to probe the shape of the droplets and cellulose partitioning in the emulsion as well as $\zeta$ potential measurements to monitor the overall surface charge of the droplets and then compared these results with rheological studies. This study sheds some light on the process of emulsion stabilization when CNF are used and especially highlights in more detail the cellulose organization at the oil/water interface. Moreover, it allowed us to probe the effect of the CNF surface charge and addition of salt on the emulsion stabilization and overall rheological properties.

\section{EXPERIMENTAL SECTION}

Materials. Oxidized cellulose nanofibrils (OCNF) were prepared by $\mathrm{TEMPO} / \mathrm{NaOCl} / \mathrm{NaBr}$ oxidation of purified softwood (spruce) fiber (resulting in a carboxylate content of $1.8 \mathrm{mmol} / \mathrm{g}$ ) and were dispersed by high-pressure homogenization to give a ca. $8 \mathrm{wt} \%$ paste, as previously reported in ref 42 . The paste was purified to remove salts and preservatives by mixing ca. $20 \mathrm{~g}$ of OCNF in $100 \mathrm{~mL}$ of deionized water (DI, $18.2 \mathrm{M} \Omega \mathrm{cm}$ ). The suspension was stirred for $30 \mathrm{~min}$ before adjusting the $\mathrm{pH}$ to $\mathrm{pH}=3$ using a $1 \mathrm{M}$ aqueous $\mathrm{HCl}$ solution. It was then dialyzed against DI (cellulose dialysis tubing MWCO 12 400) for 3 days with the DI replaced daily. The dispersion was subsequently freeze dried. Alternatively, the paste was directly freeze dried, and the purification was done after suspension preparation (see below). OCNF present a degree of oxidation measured at $22 \%$ via conductometric titration. ${ }^{6,41}$ Cationic cellulose nanofibrils (CCNF) were prepared by reaction of $\alpha$-cellulose with glycidyl trimethylammonium chloride (GTMAC) $(\geq 90 \%)$ in the presence of sodium hydroxide following an already established protocol. ${ }^{43,45}$ All components for the synthesis of $\mathrm{CCNF}$ were purchased from Sigma-Aldrich and used as received. The degree of substitution of CCNF was determined to be $23 \%$ by conductometric titration of chloride ions (trimethylammonium chloride groups) with $\mathrm{AgNO}_{3(\mathrm{aq})}$ as described previously. ${ }^{43}$ For the formation of the emulsions, hexadecane (99\%) and dodecane (99\%) were obtained from Sigma-Aldrich. For SANS measurements; fully deuterated dodecane (98\%) was purchased from CK Isotopes and used as received.

Functionalized Cellulose Nanofibril Suspension Preparation. OCNF (ca. $2 \mathrm{~g}$ ) was dispersed in $100 \mathrm{~mL}$ of water and stirred for $10 \mathrm{~min}$. The dispersion was then sonicated with a probe ultrasonicator (Vibracell VC300) using a tapered titanium microprobe $(6.5 \mathrm{~mm}$ diameter) at an intensity of $10 \mathrm{~W} \cdot \mathrm{cm}^{-2}$ (applied power determined by heat balance), alternating $1 \mathrm{~s}$ sonication with $1 \mathrm{~s}$ standby for $4 \times 15 \mathrm{~min}$. If not purified previously, the suspension was dialyzed sequentially against $0.1 \mathrm{M} \mathrm{HCl}_{(\mathrm{aq})}, 0.1 \mathrm{M} \mathrm{NaOH}_{(\mathrm{aq})}$, and $\mathrm{DI}$ water (the $\mathrm{pH}$ of the purified dispersion was 7.3).

CCNF ( 1 and $4 \mathrm{~g}$ ) were dispersed in $100 \mathrm{~mL}$ of water using an IKAT18 basic Ultra-Turrax high-speed homogenizer at $13500 \mathrm{rpm}$ for $15 \mathrm{~min}$. Hydrogels were formed by sonication using a Vibra Cell ultrasonicator (as above) at a power output of $45 \mathrm{~W} \cdot \mathrm{cm}^{-2}, 1 \mathrm{~s}$ on/off pulse for $2 \mathrm{~min}$.

Pickering Emulsion Preparation. Oil-in-water emulsions were prepared using an oil:aqueous phase volume ratio of 30:70. Typically, $0.3 \mathrm{~mL}$ of hexadecane (or dodecane for SANS studies) was mixed with 
$0.7 \mathrm{~mL}$ of OCNF or CCNF aqueous dispersion (prepared as described above) and sonicated to form the emulsion using a Vibracell VC300 using a $3 \mathrm{~mm}$ diameter tapered titanium tip at an intensity of $17 \mathrm{~W} \cdot \mathrm{cm}^{-2}$ applied power, determined by heat balance, alternating $1 \mathrm{~s}$ sonication with $1 \mathrm{~s}$ standby for $15 \mathrm{~s}$ or $2 \mathrm{~min}$. Emulsion stability, phase separation, and creaming were evaluated for emulsions prepared using different concentrations of OCNF or CCNF by observation after 1 day or by centrifuging the emulsion at $4000 \mathrm{rpm}$ for $30 \mathrm{~min}$.

Characterization. Transmission electron microscopy (TEM) images of functionalized CNF were obtained on a JEM-2100 Plus (JEOL, USA) at an operating voltage of $200 \mathrm{kV}$. Before image acquisition, samples were deposited on a copper grid and negatively stained with uranyl acetate.

The freeze-dried emulsion ( 30 vol \% cyclohexane and 70 vol \% $8 \mathrm{~g} / \mathrm{L}$ OCNF suspension) morphology was characterized using a scanning electron microscope (JEOL SEM6480LV or JEOL FESEM6301F, Japan) equipped with an energy-dispersive $\mathrm{X}$-ray spectrometer (Oxford INCA X-ray analyzer, UK) operating at an accelerating voltage of 5 or $10 \mathrm{kV}$. Samples were coated with chromium or gold.

Emulsion droplet sizes (Sauter diameter $D(3,2)$ ) were characterized using a Mastersizer X laser diffraction particle size analyzer (Malvern, UK) equipped with optics appropriate for detection of particle sizes in the $0.5-180 \mu \mathrm{m}$ range. Samples were measured in triplicate.

Water and hexadecane contact angles on spin-coated films of OCNF, produced from dispersions at $\mathrm{pH} 7.3$ deposited and dried on glass slides, were determined using the sessile drop method at room temperature. Static images were captured using a Discovery VMS-001 USB microscope (Veho) and Dropsnake software with the ImageJ imaging process package to analyze images. Each measurement was conducted in triplicate.

$\zeta$-potential measurements of CNF dispersions and CNF-stabilized emulsions at $10 \mathrm{~g} / \mathrm{L} \mathrm{CNF}$ and $0,0.1$, or $0.5 \mathrm{M} \mathrm{NaCl}$ in the water phase were also conducted using a Malvern Zetasizer Nano ZSP (Malvern, UK). The dispersions and emulsions were diluted 10 times using the corresponding salt buffer. The $\zeta$ potential was averaged over 7 measurements of 100 scans and repeated 4 times.

Rheology experiments were conducted on a Discovery HR-3 (TA Instruments) in plate-plate geometry (40 $\mathrm{mm}$ diameter). The geometry was covered with a thin layer of mineral oil to avoid evaporation. Oscillatory strain experiments were conducted at $25^{\circ} \mathrm{C}$. A preconditioning step of $20 \mathrm{~s}$ at zero shear was applied before each measurement. Oscillatory frequency experiments were conducted at a strain of $0.5 \%$ within the linear viscoelastic regime. Finally, steady flow measurements were carried out for a shear rate ranging from $10^{-1}$ to $10^{2}$ $\mathrm{s}^{-1}$.

Emulsion droplets were also studied using an inverted confocal laser scanning microscope (CLSM) (ZEISS LSM 880, Germany) in the resonant mode with a $100 \times$ oil immersion objective. The emulsion suspension $(6 \mu \mathrm{L})$ was sandwiched between the coverslips and hermetically sealed by a $120 \mu \mathrm{m}$ thick spacer with a $5.1 \mathrm{~mm}$ aperture (SecureSeal Imaging). Before adding the solution, typically $40 \mu \mathrm{L}$ of calcofluor white (from Sigma-Aldrich) was added to $1 \mathrm{~mL}$ of solution to allow imaging of the cellulose shell, since this dye fluoresces only when bonded to the cellulose nanofibrils. ${ }^{46}$

Small-angle neutron scattering (SANS) experiments were carried out at the Sans2d instrument at ISIS, Rutherford Appleton Laboratory, UK. The $q$ range of the measurements was from 0.0015 to $0.25 \AA^{-1}$, and all experiments were conducted at $25{ }^{\circ} \mathrm{C}$. SANS with contrast variation was employed as a noninvasive technique to obtain structural information from the samples in situ based on scattering length density (SLD) differences (see Table S1, Supporting Information). Data were collected for five different sets of hydrogen- and deuterium-bearing components (for OCNF and CCNF, both hydrogenated): h-oil/ $\mathrm{D}_{2} \mathrm{O}$ and $\mathrm{d}$-oil $/ \mathrm{H}_{2} \mathrm{O}$ give a full core-shell contrast for the droplets, d-oil/ $\mathrm{D}_{2} \mathrm{O}$ allows scattering from the cellulose only, while h-oil $/ 50 \% \mathrm{D}_{2} \mathrm{O}$ is used to see the core of the droplets only. A last contrast, $h$-oil $/ 70 \% \mathrm{D}_{2} \mathrm{O}$, was used to confirm results obtained by the other contrasts. The samples were prepared less than 1 week prior to the SANS experiment. If creaming was observed, the upper phase was selected and pipetted into a $1 \mathrm{~mm}$ thick SANS cell. All of the SANS patterns acquired were checked for multiple scattering.

The scattering intensity $I(q)$ of the cellulose nanofibrils suspensions can be described as

$$
I_{\mathrm{CNF}}(q)=K P_{\text {cyl }}\left(q, R_{\max }, \varepsilon, L, b_{\text {Kuhn }}\right)+B_{g}
$$

where $K$ is the scattering factor, $P_{\text {cyl }}\left(q, R_{\max } \varepsilon, L, b_{\mathrm{Kuhn}}\right)$ is the form factor for flexible rods with an elliptical cross-section, taking into account the major radius of the cross section $R_{\max }$ the cross-section ellipticity $\varepsilon$, the fibril overall length $L$, and the Kuhn length $b_{\text {Kuhn }} \leq L$ ( 2 times the persistence length), and $B_{g}$ is a background parameter. For rigid rods, $b_{\text {Kuhn }}=L$. We previously suggested that the Kuhn length could be used qualitatively as an indication of the mesh size in a 3D network made of cellulose nanofibrils. ${ }^{42}$ No structure factor describing fibril-fibril interactions was required. A detailed description of this model and its use to describe CNF can be found in a report by Schmitt et al. ${ }^{42}$

Emulsion droplets were fitted as polydisperse core-shell spheres using the onion model. ${ }^{47}$ The core of the sphere corresponds to the oil droplet, while the shell is associated with the cellulose layer (see Scheme 1)

$$
I_{\text {drop }}(q)=\left\langle K P_{\text {sph }}\left(q, R_{c}, t_{\text {shell }}, \rho_{\text {core }}, \rho_{\text {shell }}(A), \rho_{0}\right)\right\rangle_{\sigma}+B_{g}
$$

\section{Scheme 1. Sketch of a Droplet of an Oil-in-Water Emulsion ${ }^{a}$}

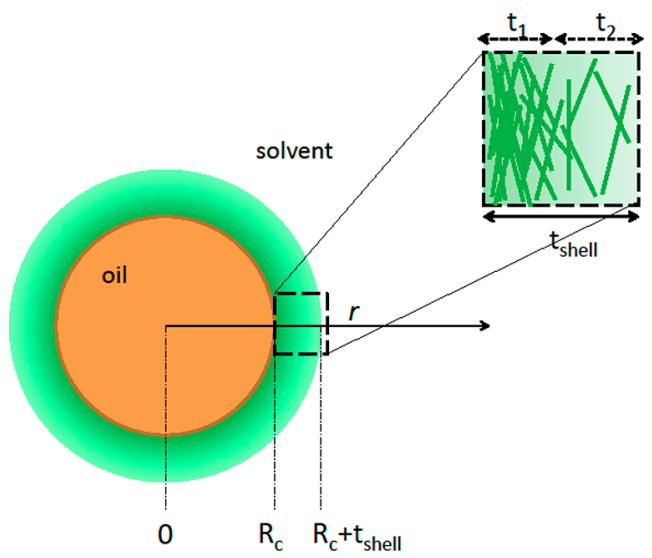

${ }^{a}$ The droplets are modeled as core-shell spheres with a core radius $R_{c}$ and a shell thickness $t_{\text {shell. }}$. The core is made of oil, while the shell is made of cellulose in water. The shell presents a variation of hydration from fully packed cellulose nanofibrils to a fully hydrated shell (inset). $t_{1}$ is the thickness associated with fully packed CNF, and $t_{2}$ is where the hydration cannot be neglected. $t_{1}$ and $t_{2}$ are arbitrarily found from the SLD profile reconstruction.

where $K$ is the scaling factor and $P_{\text {sph }}\left(q, R_{\mathcal{c}} t_{\text {shell }}, \rho_{\text {core }} \rho_{\text {shell }}, \rho_{0}\right)$ is the form factor of the core-shell sphere with a core radius $R_{\mathrm{c}}$ (fixed at $1 \mu \mathrm{m}$, outside the measurable $q$ range) and shell thickness $t_{\text {shell }}$ (in nm). $\rho_{\text {shell }}$, $\rho_{\text {core }}$ and $\rho_{0}$ are the scattering length densities (SLD) of the droplet shell, the oil core, and the solvent, respectively. $\rho_{\text {core }}$ (oil) and $\rho_{0}$ (water) are known for each contrast studied (see Table S1, Supporting Information).

The shell was modeled as a thick shell with densely packed cellulose nanofibrils at the oil/water interface $\left(r=R_{c}\right)$ and a nonlinear increase of hydration until it becomes fully hydrated at $R_{\mathrm{c}}+t_{\text {shell }}$ (see Scheme 1 ). To reproduce this profile, the SLD of the shell is described as

$$
\rho_{\text {shell }}(A, r)=B e^{A\left(r-R_{\mathrm{c}}\right) / t_{\text {shell }}}+C \text { for } R_{\mathrm{c}} \leq r \leq R_{\mathrm{c}}+t_{\text {shell }}
$$

where $A$ is the decay rate of the shell, adjusted during the fitting procedure, and $B$ and $C$ are constants, so $\rho_{\text {shell }}\left(A, R_{c}\right)=\rho_{\text {cell }}$ and $\rho_{\text {shell }}\left(A, R_{c}+t_{\text {shell }}\right)=\rho_{0}$ with $\rho_{\text {cell }}=2.42 \times 10^{-6} \AA^{-2}$, the SLD of cellulose, obtained via contrast variation studies of OCNF suspensions in different $\mathrm{D}_{2} \mathrm{O} / \mathrm{H}_{2} \mathrm{O}$ solvents (see Figure $\mathrm{S} 1$, Supporting Information). Note that theoretical calculation of $\rho_{\text {cell }}$ would give $1.75 \times 10^{-6} \AA^{-2}$. 
The difference can be explained by the presence of 3 labile hydrogens in the chemical formula of cellulose that can be exchanged with deuterium present in the solvent or oil. A nonlinear hydration of the shell is hence given by the parameter $A$, which allows the reconstruction of the scattering length density profile along the droplet radius. Note that in most cases, the density profiles of the shells obtained can be arbitrarily cut in two regions: one of thickness $t_{1}$, where the SLD corresponds to densely packed cellulose, and another one $t_{2}$, where the effect of hydration is obvious in the profile $\left(t_{\text {shell }}=t_{1}+t_{2}\right.$, see Scheme 1$)$. Finally, the polydispersity in the droplet size is taken into account using the Gaussian distribution, and the polydispersity index for both oil core and shell thickness was fixed at $20 \%$ for all emulsions. $B_{g}$ is a background parameter. Models for polydisperse core-shell spheres are described, for example, in Manet et al., ${ }^{48}$ and more details are provided in the Supporting Information.

Finally, for the $\mathrm{d}$-oil $/ \mathrm{D}_{2} \mathrm{O}$ contrast where the oil core is hidden, patterns were fitted by the sum of $I_{\mathrm{CNF}}(q)$ and $I_{\text {drop }}(q)$. In that case, $\rho_{\text {core }}$ $\approx \rho_{0}$ and only the cellulose shell is observed. No hydration of the shell was taken into account to fit this contrast (parameter $A$ fixed at $A=0$; hence, $\left.\rho_{\text {shell }}(r)=\rho_{\text {cell }}\right)$ to avoid overparametrization. In that case, parameters of the fibrils $\left(R_{\max }, \varepsilon, L\right)$ and of the droplets $\left(R_{\mathcal{c}}, t_{\text {shell }}\right)$ were fixed in agreement with results from suspensions and other contrasts, and only the two scaling factors and the Kuhn length $b_{\text {Kuhn }}$ were left free.

\section{RESULTS AND DISCUSSION}

Characterization of the CNF Used. Both OCNF and CCNF were observed in TEM and can be described as rod-like particles (Figure 1a and $1 \mathrm{~b}$ ) with a typical length and cross-
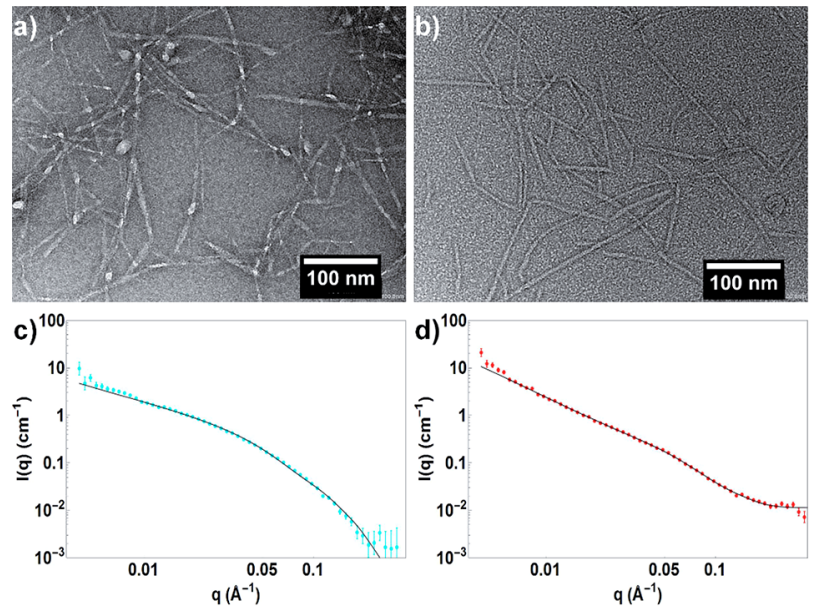

Figure 1. TEM micrographs of (a) OCNF and (b) CCNF after dispersion in water at $0.025 \mathrm{wt} \%$. Staining obtained using uranyl acetate. SANS patterns of (c) OCNF and (d) CCNF at $10 \mathrm{~g} / \mathrm{L}$ in $\mathrm{D}_{2} \mathrm{O}$. Data were fitted with the model of rigid rods with an elliptical cross section.

section diameter obtained from measurements of over 100 particles of $L=160 \pm 60 \mathrm{~nm}$ and $D=7 \pm 2 \mathrm{~nm}$ for OCNF and $L$ $=105 \pm 35 \mathrm{~nm}$ and $D=7 \pm 2 \mathrm{~nm}$ for CCNF (length and diameter size distributions can be found in ref 42 and in Figure S2, Supporting Information, for OCNF and CCNF, respectively).

SANS data (Figure 1c and 1d) were modeled using a rod-like model with an elliptical cross-section. Cross section radii $R_{\max }$ and $R_{\min }$ were found to be $5.0 \pm 0.1$ and $1.1 \pm 0.1 \mathrm{~nm}$ for OCNF and $4.9 \pm 0.1$ and $1.3 \pm 0.1 \mathrm{~nm}$ for CCNF, respectively. The length $L$ is in both cases above $100 \mathrm{~nm}$ and cannot be probed in the used $q$ range. For OCNF, the rod-like particles are rigid with a Kuhn length $b_{\text {Kuhn }}$ above $100 \mathrm{~nm}$. For CCNF, the particles are seen as semiflexible with $b_{\text {Kuhn }}=13.8 \pm 0.1 \mathrm{~nm} \ll L$. The results are consistent with the TEM imaging and with values reported previously for both $\mathrm{OCNF}^{6,49}$ and CCNF. ${ }^{43,45}$

The calculation of a contact angle of $39^{\circ}$ for OCNF at the hexadecane/water interface using Young's equation (Figure S3) confirmed that an an $\mathrm{o} / \mathrm{w}$ emulsion could be formed in the presence of OCNF. ${ }^{19}$

Emulsion Formulation: Stability and Droplet Size. Oilin-water emulsions were readily formed upon sonication of mixtures of the selected oil and OCNF or CCNF aqueous dispersions. With OCNF, the formed emulsions were relatively insensitive to changes in $\mathrm{pH}$, the droplet size remaining stable over the $\mathrm{pH}$ range tested: $3-11.5$ (Figure S4a).

The effect of OCNF concentration on the emulsion droplet size and stability was assessed in the range $0-15 \mathrm{~g} / \mathrm{L}$, see Figure 2 a. Increasing the OCNF concentration induces a decrease of

\section{a)}

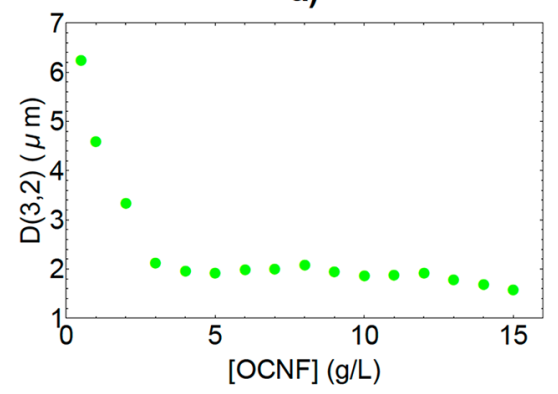

b)

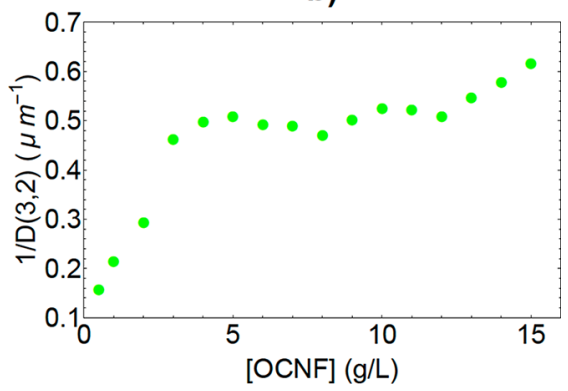

Figure 2. (a) Sauter diameter $D(3,2)$ of the droplets of hexadecane-inwater emulsions stabilized by OCNF, measured as a function of OCNF concentration in the aqueous phase. (b) Same data given as $1 / D(3,2)$. Error bars, obtained from triplicates, are smaller than the markers.

the droplet size until a plateau is reached for ca. $3 \mathrm{~g} / \mathrm{L}$. Plotting $1 / D(3,2)$ versus concentration (Figure $2 b$ ) displays the size variations in more detail. A sharp increase of $1 / D(3,2)$ in the low-concentration range is observed due to the formation of a stable shell of particles at the droplet interface, before a much weaker increase, when more fibrils are added, once the shell is complete. These results follow the typical behavior for Pickering emulsions ${ }^{33}$ and indicate that OCNF act as a true Pickering agent, where for concentrations above $3 \mathrm{~g} / \mathrm{L}$ the surface of the droplets is well covered and the interfacial tension cannot be altered by adding more particles, resulting in a stable droplet size.

The size of the droplets is found to be ca. $2 \mu \mathrm{m}$ for emulsions containing more than $3 \mathrm{~g} / \mathrm{L}$ OCNF in the aqueous phase. At lower concentrations of OCNF, the droplets are not efficiently stabilized, inducing some coalescence and phase separation. This is confirmed by the complete phase separation observed after centrifugation at $4000 \mathrm{rpm}$ for $30 \mathrm{~min}$ of the emulsion with OCNF concentration $\leq 3 \mathrm{~g} / \mathrm{L}$. For higher fibril concentrations, 
centrifugation results in a creaming phenomenon, concentrating the droplets in the upper phase but without phase separation, suggesting strong adsorption of OCNF at the o/w interface. Prior to centrifugation, creaming is observed after 1 day only for concentrations $\leq 8 \mathrm{~g} / \mathrm{L}$, while stable emulsions are formed above this concentration (see Figure S5).

As salt is known to affect the rheology of OCNF dispersions, the effect of $\mathrm{NaCl}$ concentration on the droplet size was investigated. Addition of salt resulted in a decrease of the OCNF concentration needed to stabilize o/w droplets (see Figure S6, phase separation after centrifugation is found only for OCNF concentration $\leq 0.5 \mathrm{~g} / \mathrm{L}$ at $1 \mathrm{M} \mathrm{NaCl}$ ), in agreement with what has been observed for $\mathrm{CNC}^{14}$ On the other hand, the size of the emulsion droplets is found to drastically increase when the concentration of OCNF is decreased in the presence of salt (see Figure 3). Samples at $0.1 \mathrm{M}$ showed a slightly different behavior

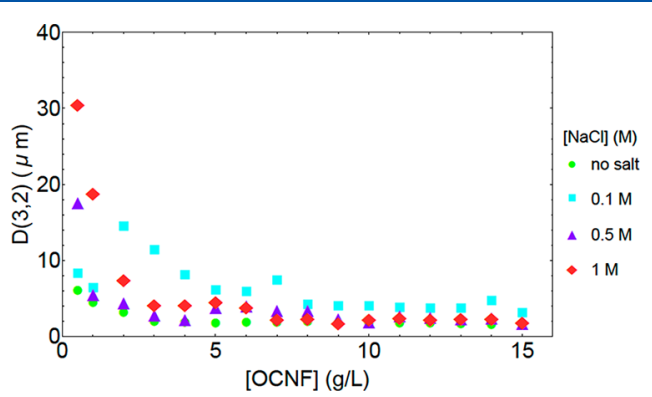

Figure 3. Droplet Sauter diameter $D(3,2)$ measured for hexadecane-inwater emulsions stabilized by OCNF as a function of OCNF concentration in the aqueous phase and for salt $(\mathrm{NaCl})$ concentrations ranging from 0 to $1 \mathrm{M}$. Error bars, obtained from triplicates, are smaller than the markers.

than those at other salt concentrations with higher droplet sizes compared to other salt amounts and a more pronounced decrease of the droplet diameter with OCNF concentration above $8 \mathrm{~g} / \mathrm{L}$. Nonetheless, for all $\mathrm{NaCl}$ concentrations, no creaming and little variation in the droplet size are found for OCNF concentrations higher than $8 \mathrm{~g} / \mathrm{L}$. Moreover, all samples containing salt show a self-standing gel behavior (pictures upside down in Figure S6), while they are much more fluid without salt (pictures not shown). This indicates the formation of a percolating network between droplets stabilizing the emulsion, consistent with results from Gestranius et al. ${ }^{27}$

Having obtained well-stabilized emulsions at $10 \mathrm{~g} / \mathrm{L}$ of OCNF in the aqueous phase, similar Pickering emulsions were prepared using $\mathrm{CCNF}$ at the same concentration, and the droplet diameter was observed to be ca. $2 \mu \mathrm{m}$ in that case. These emulsions showed a slight size variation with $\mathrm{pH}$ (see Figure $\mathrm{S} 4 \mathrm{~b})$ that can probably be related to the decrease of the $\zeta$ potential of CCNF with increasing $\mathrm{pH}^{50}$ while the OCNF $\zeta$ potential does not vary in the $\mathrm{pH}$ range tested. ${ }^{51}$ When salt was added $(0.1$ and $0.5 \mathrm{M})$, no visual change was observed for these samples, since they were still flowing.

Characterization of the Shell: Ex Situ Studies. To evidence the role of CNF as Pickering agent in the stabilization of emulsions, we prepared an emulsion using OCNF at $8 \mathrm{~g} / \mathrm{L}$ and replaced hexadecane with a more volatile oil, cyclohexane, without salt. After freeze drying, the obtained structures reflect the OCNF boundaries at the $\mathrm{o} / \mathrm{w}$ interface as observed using SEM, although the freeze-drying process collapses the OCNF network into denser structures than are present in solution (Figure 4a).
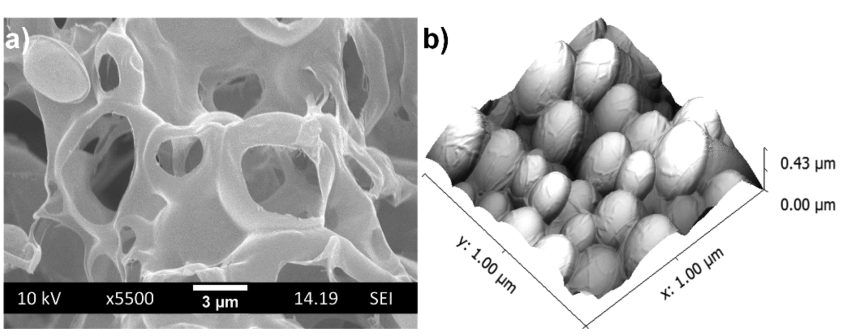

Figure 4. (a) SEM micrograph of the structures remaining after freeze drying a cyclohexane-in-water emulsion stabilized by OCNF. (b) AFM image of polymerized styrene/water emulsion stabilized by OCNF.

Similarly, AFM was used to examine the structures remaining after an emulsion of styrene in water stabilized by OCNF was polymerized to "set" the droplets as polystyrene (Figure $4 \mathrm{~b}$ ). ${ }^{22,52}$ Droplet sizes are found in that case to be much smaller than those for hexadecane, probably due to the oil change and droplet volume decrease during the polymerization process.

Both results clearly suggest the presence of OCNF at the interface of the droplets, ensuring the stability of the emulsion and acting as Pickering agents, in agreement with the droplet size studies.

Characterization of the Shell: In Situ Studies. Although both SEM and AFM data support the assertion that CNF stabilize emulsions by acting as Pickering particles in a similar fashion to cellulose nanocrystals, ${ }^{21}$ such measurements require severe perturbation of the system by drying or polymerization. Thus, to have a better depiction of the emulsions and the organization of the nanofibrils, samples were studied using CLSM and SANS.

CLSM was used to study the cellulose distribution in OCNFstabilized emulsions at $10 \mathrm{~g} / \mathrm{L}$ with and without salt (see Figures 5 and S7). Fluorescent staining using calcofluor white binding to the cellulose allows clear identification of the cellulose shell. Without salt, droplets are found well separated and little to no staining of the water phase is observed, indicating that most of OCNF is used as a Pickering agent, residing in the shell around
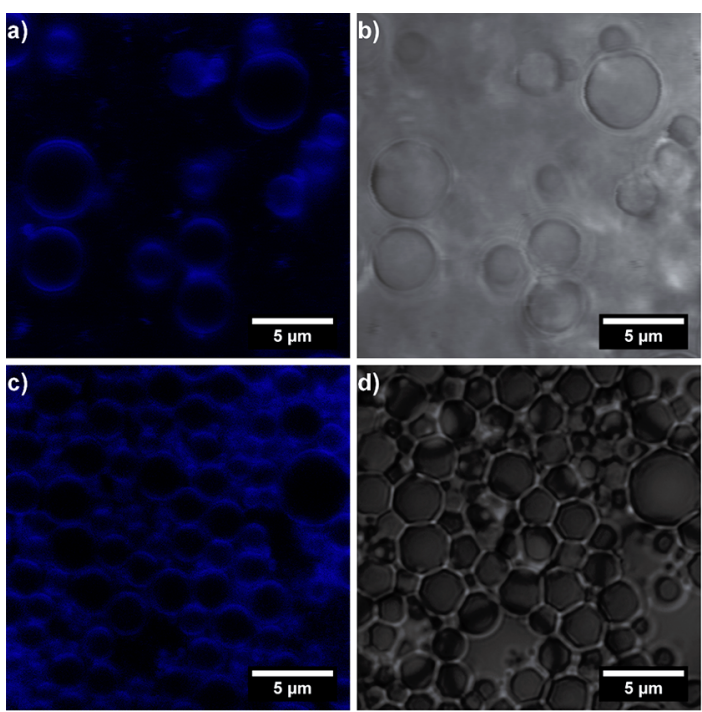

Figure 5. CLSM micrographs of hexadecane-in-water emulsion at $10 \mathrm{~g} /$ L OCNF in the water phase: ( $a$ and b) without salt and ( $c$ and d) with $0.1 \mathrm{M} \mathrm{NaCl}$. Each image is given in (a and c) the fluorescent channel and ( $b$ and $d$ ) the bright-field channel. Calcofluor white was used as dye. 
the oil droplets. Addition of salt drastically changes the system with the formation of aggregates of droplets, in agreement with previous studies ${ }^{40}$ and visual observations (Figure S5). The concentration of the salt has an influence on the aggregates formed with a large network entrapping droplets at $0.1 \mathrm{M}$, while at $0.5 \mathrm{M} \mathrm{NaCl}$ cellulose forms rough shells around aggregating droplets (Figure S7).

SANS measurements were carried out using hexadecane or dodecane as the oil to probe the cellulose shell in more detail. The choice of oil had little influence on the SANS patterns and hence on the cellulose shell (see Figure S8); hence, dodecane was used to allow contrast variation techniques using a deuterated version of the oil.

Emulsions of dodecane in $\mathrm{D}_{2} \mathrm{O}$ stabilized by OCNF at $10 \mathrm{~g} / \mathrm{L}$ without salt were studied at five different contrasts (see Figure 6a).
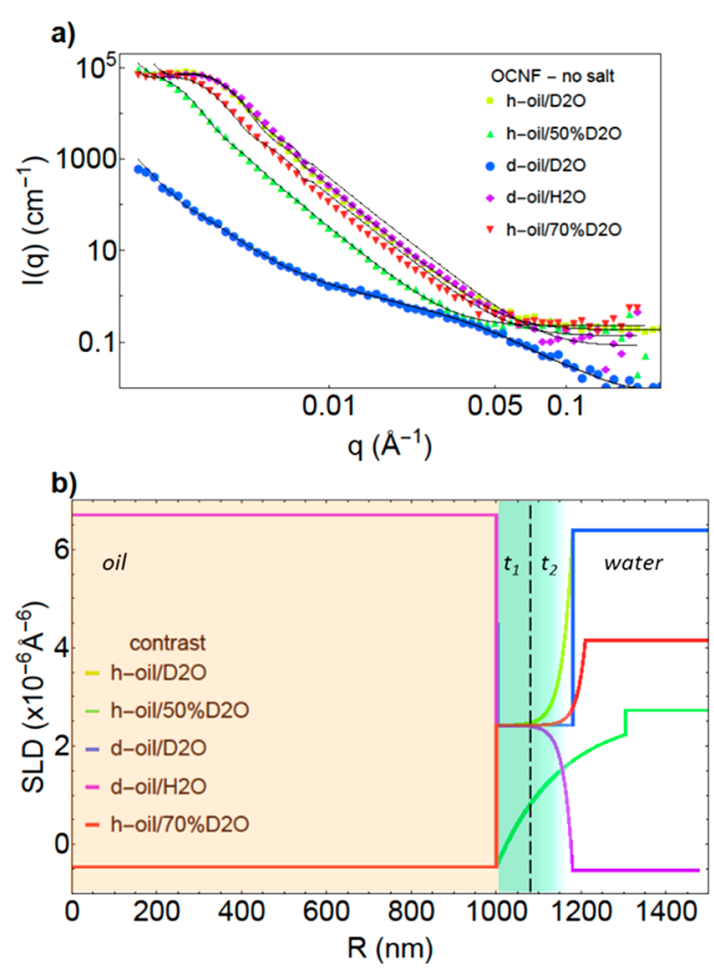

Figure 6. (a) Experimental SANS patterns of a dodecane/water emulsion stabilized by OCNF $(10 \mathrm{~g} / \mathrm{L}$ in the water phase) without salt for 5 different contrasts. (Black) Fits made using the onion model (four contrasts) and a mixture of core-shell spheres and OCNF fibrils (d-oil/ $\mathrm{D}_{2} \mathrm{O}$ ). (b) SLD profiles of the droplets obtained from the fits. Oil core and cellulose-rich shell (with regions $t_{1}$ and $t_{2}$ ) are evidenced by a colored background to guide the eye (see Scheme 1).

For h-oil $/ \mathrm{D}_{2} \mathrm{O}$, d-oil $/ \mathrm{H}_{2} \mathrm{O}$, h-oil $/ 50 \% \mathrm{D}_{2} \mathrm{O}$, and h-oil/70\% $\mathrm{D}_{2} \mathrm{O}$ contrasts, the patterns show an oscillation at small angles that we associate with the shell thickness and a decrease in intensity linked to the shell-water interface, while for d-oil/ $\mathrm{D}_{2} \mathrm{O}$, the signal resembles the pattern of OCNF dispersions with an increase at small angles due to the arrangement of OCNF within the shell. The four first contrasts were fitted using the core-shell onion model described in the Experimental Section, while the last one was fitted using the combination of a signal for OCNF fibrils (with parameters fixed according to results given by Figure 1, except for the Kuhn length $b_{\text {Kuhn }}$ ) plus a shell sphere (core and water contrast matched and shell thickness fixed according to other contrasts). These fitting procedures allow the scattering length density (SLD) profiles along the radius of the droplets to be obtained for each contrast, as shown in Figure $6 \mathrm{~b}$ (values found from the fitting procedure are given in Table S2, Supporting Information).

The results show that the droplets have a dense and thick cellulose shell with a shell thickness $t_{\text {shell }}$ typically reaching 200 $\mathrm{nm}$, much larger than the OCNF cross-section or the thickness found in the case where CNC are used as Pickering agents. ${ }^{33}$ In all SLD profiles, this shell thickness presents two regions $t_{1}$ and $t_{2}$. The first region, $t_{1}$ of ca. $100 \mathrm{~nm}$, has a SLD equal to $\rho_{\text {cell }}$ the SLD of cellulose, and corresponds to a layer of densely packed CNF, while the second region $t_{2} \approx 100 \mathrm{~nm}$ where the SLD decays from $\rho_{\text {cell }}$ toward $\rho_{0}$ (the SLD of the solvent) represents a hydration layer between cellulose and water (see Scheme 1). For the $\mathrm{d}$-oil $/ \mathrm{D}_{2} \mathrm{O}$ contrast, the hydration of the shell is not included in the fit to avoid over-parametrization $\left(t_{1}=t_{\text {shell }} \approx 200 \mathrm{~nm}\right)$.

Without salt, the contribution of OCNF for the $d$-oil $/ \mathrm{D}_{2} \mathrm{O}$ contrast can be fitted similarly to the signal of OCNF in suspensions without any extra interactions or change in the Kuhn length (rigid rods), indicating OCNF in the shell are not experiencing strong repulsion.

SLD profiles of the shell are consistent for all contrasts with the exception of h-oil $/ 50 \% \mathrm{D}_{2} \mathrm{O}$. This last contrast was chosen to hide the signal of cellulose; nonetheless, a large shell is still obtained but with a SLD between the value for hydrogenated dodecane and cellulose, probably signaling that part of the cellulose is present in the oil phase. Nonetheless, caution should be taken concerning the level of detail obtained from the fits. Indeed, an important portion of the scattering signal of the droplets is at lower $q$ and so not covered in the accessible $q$ range, which prevents a complete modeling and hence induces some uncertainty in the interpretation of the data.

Similar results are obtained for emulsions prepared with 0.1 and $0.5 \mathrm{M}$ salt with little difference in the size and hydration of the shell (see Figure S9 and Table S2 for the values found from the fits, especially the values of $\left.t_{\text {shell }}\right)$. The main difference observed concerns the $\mathrm{d}$-oil $/ \mathrm{D}_{2} \mathrm{O}$ contrast, where this time the Kuhn length associated with the signal of the fibrils ( $>100 \mathrm{~nm}$ for the pattern without salt) decreases to $27.0 \pm 0.1 \mathrm{~nm}$ for $[\mathrm{NaCl}]$ $=0.1 \mathrm{M}$ and $15.5 \pm 0.1 \mathrm{~nm}$ for $[\mathrm{NaCl}]=0.5 \mathrm{M}$. A similar trend was observed for dispersions of OCNF in the presence of salt, and rather than an actual change in the flexibility of the fibrils with salt, this is attributed to the signature of fibril aggregation into a 3D network since SAXS or SANS cannot distinguish between junctions between overlapping fibers and a bend in a single fiber. ${ }^{42}$ This aggregation of fibrils in the shell with the addition of salt could explain the CLSM observations with a 3D network spanning between shells, inducing aggregation of the droplets. It was shown previously that for dispersions this aggregation in the presence of salt was associated with charge screening between OCNF. ${ }^{42}$

By comparison, CCNF-stabilized emulsions without salt showed again the presence of cellulose at the droplet interfaces in CLSM, while SLD profiles extracted from the fitting of the SANS data evidence again a thick shell (see Figure 7). SANS data and fits acquired for these emulsions without and with salt are presented in Figure S10, while parameters from the fits are in Table S3. One can observe a larger shell for the emulsions without salt compared to the samples with salt $\left(t_{\text {shell }}\right.$ ca. 200 versus $170 \mathrm{~nm}$ taking into account the hydration layer), indicating a slightly more compact shell with CCNF compared to OCNF. 


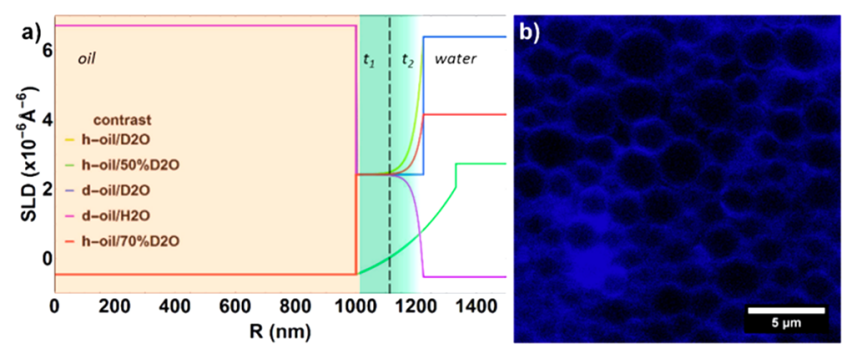

Figure 7. (a) SLD profiles of the droplets obtained from fitting the SANS data of a dodecane/water emulsion stabilized by CCNF $(10 \mathrm{~g} / \mathrm{L}$ in the water phase) without salt for 5 different contrasts. SANS patterns and fits can be found in Figure S10a. Oil core and cellulose-rich shell (with regions $t_{1}$ and $t_{2}$ ) are evidenced by a colored background to guide the eye (see Scheme 1). (b) CLSM micrograph of the same type of emulsion. Calcofluor white was used as dye.

Contrary to OCNF, CCNF are modeled as semiflexible even without salt $\left(b_{\mathrm{Kuhn}}=13.8 \pm 0.1 \mathrm{~nm} \ll L\right)$, which can be attributed to the greater apparent flexibility of these fibrils. No variation of $b_{\text {Kuhn }}$ is required to fit the data in the presence of salt.

It is clear from CLSM studies that cellulose nanofibrils, both OCNF and CCNF, act as Pickering agents, forming a clear shell around the oil droplets with little CNF isolated in the water phase. Interestingly, analysis of the SANS data pinpoint an unusual behavior as the CNF do not form a single monolayer at the interface but a thick shell made of a CNF network which becomes more and more hydrated as we move away from the oil/water interface.

Addition of salt to OCNF-stabilized emulsions induces a change in the OCNF Kuhn length, which may be an indication of an aggregation of the fibrils within the shell. This would in turn induce aggregation between the droplets and explain the CLSM observations. On the other hand, CCNF does not evidence a change in conformation within the shell (although a thinner shell is observed in the presence of salt). To understand these observations, we studied the effect of the addition of salt on the droplet surface charge.

Effect of Salt: Droplet Surface Charge. Since OCNF bear negative charges, ${ }^{42,51}$ emulsion droplets stabilized by OCNF are assumed to experience electrostatic repulsion with other droplets, ${ }^{27}$ which is progressively screened by the addition of salt, explaining the aggregation results obtained from CLSM. Hence, $\zeta$-potential measurements were carried out on OCNFand CCNF-based emulsions at $10 \mathrm{~g} / \mathrm{L}$ in the water phase and at different salt concentrations, and the results were compared with the $\zeta$-potential measurements from the corresponding dispersions (see Table 1).

For both OCNF dispersions and emulsions, the addition of $\mathrm{NaCl}$ results in lowering the surface charge, ranging from -45 $\mathrm{mV}$ at $0 \mathrm{M}$ to $-7 \mathrm{mV}$ at $0.5 \mathrm{M}$ for dispersions (in agreement with previous studies ${ }^{42,53}$ ) and from -46 to $-5 \mathrm{mV}$ for emulsions. A value of $-30 \mathrm{mV}$ is often considered as the limit of stability for

Table 1. $\zeta$-Potential (in $\mathrm{mV}$ ) Obtained for Dispersions of CNF and CNF-Stabilized Emulsions

\begin{tabular}{cccccc} 
& \multicolumn{2}{c}{ OCNF } & & \multicolumn{2}{c}{ CCNF } \\
\cline { 2 - 3 } \cline { 5 - 6 }$[\mathrm{NaCl}](\mathrm{M})$ & dispersions & emulsions & & dispersions & emulsions \\
0 & $-45 \pm 5$ & $-46 \pm 11$ & & $37 \pm 6$ & $24 \pm 7$ \\
0.1 & $-22 \pm 5$ & $-19 \pm 1$ & & $17 \pm 2$ & $20 \pm 1$ \\
0.5 & $-7 \pm 3$ & $-5 \pm 1$ & & $7 \pm 4$ & $13 \pm 3$
\end{tabular}

dispersions of colloids by electrostatic repulsion; hence, in both cases the systems will experience aggregation for $\mathrm{NaCl} \geq 0.1 \mathrm{M}$, resulting in droplet aggregation for the emulsion. An increase in the salt amount results in a higher attraction between droplets and ultimately a smaller droplet-droplet distance. This causes a faster gelation, preventing a dense packing, resulting in a rougher droplet interface, as observed in the CLSM micrographs. CCNF dispersions and CCNF-based emulsions also experience changes in their $\zeta$-potential, ranging from 37 to $7 \mathrm{mV}$ for dispersions and from 24 to $13 \mathrm{mV}$ for emulsions. Nonetheless, it can be noted that for emulsions the change in $\zeta$-potential is much weaker and that even at $0 \mathrm{M}$ salt its value is found to be lower than the $30 \mathrm{mV}$ needed to prevent aggregation. The weak change in $\zeta$-potential hence explains the lower change in the visual observations on those samples.

CLSM, SANS, and $\zeta$-potential results hence indicated that the OCNF-stabilized emulsion droplets show a clear sensitivity to the addition of salt, while CCNF-based emulsions are less sensitive to salt. Those behaviors are expected to strongly influence the rheological properties of the emulsions.

Effect of Salt: Rheology of the Emulsions. Rheological studies were performed on freshly prepared OCNF dispersions and OCNF-stabilized emulsions at $10 \mathrm{~g} / \mathrm{L}$ and their CCNF counterparts.

Viscosity measurements with shear (see Figure 8) showed shear-thinning properties for all of the samples. Without salt, the

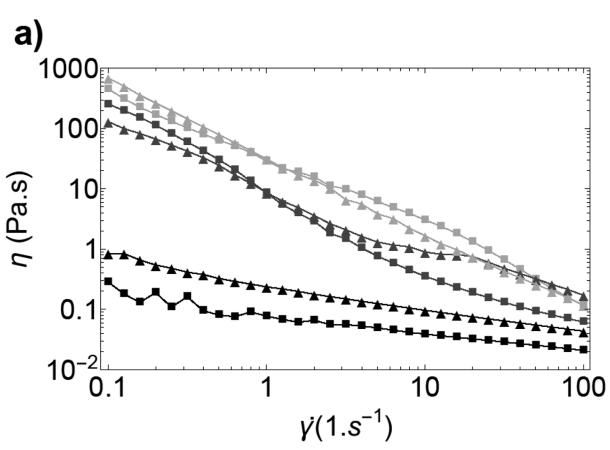

b)

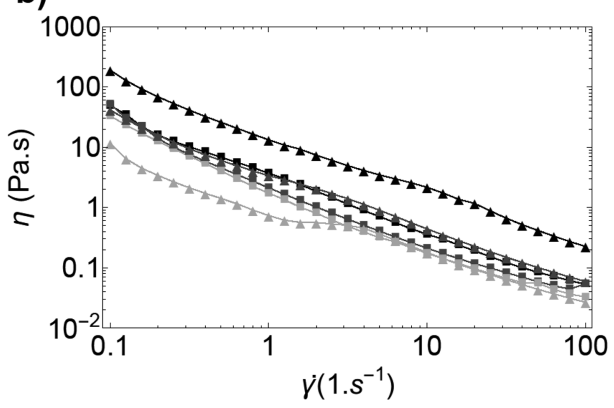

Figure 8. Shear viscosity measurements of (a) OCNF and (b) CCNF dispersions (square symbols) and emulsions (triangles) at $10 \mathrm{~g} / \mathrm{L}$ of $\mathrm{CNF}$ in the water phase with various amounts of $\mathrm{NaCl}$ : (black) 0 , (dark gray) 0.1 , and (pale gray) $0.5 \mathrm{M} \mathrm{NaCl}$.

OCNF-based emulsion presents a similar viscosity trend to that of its dispersion counterpart; whereas the viscosity is highly increased for CCNF emulsions. This may be related to the difference of $\zeta$-potentials between the CCNF dispersion and the emulsion. Indeed, the $\zeta$-potential of the CCNF-stabilized droplets is found to be below $30 \mathrm{mV}$, while it is above $30 \mathrm{mV}$ 
for the CCNF dispersion; hence, aggregation is already more pronounced for the emulsions in that case.

When salt is added, the viscosity strongly increases for OCNF systems (from 1 to almost $1000 \mathrm{~Pa} \cdot \mathrm{s}$ at $\dot{\gamma}=0.1 \mathrm{~s}^{-1}$ when salt concentration is varied from 0 to $0.5 \mathrm{M}$ ) and dispersions and emulsions present the same viscosity curves. This is crucial as it shows that although most of the fibrils are present within the shell around the emulsion droplets (as evidenced in CLSM and SANS), the behavior and aggregation within and between the droplet shells drives the rheological properties. For CCNF, little variation is observed with salt (except a slight decrease of the viscosity between $0 \mathrm{M}$ and the other salt amounts, which can be related to the decrease of the shell thickness observed in SANS).

Oscillatory measurements (frequency sweeps in Figures S11 and S12 and amplitude sweeps in Figures 9 and S13) were also

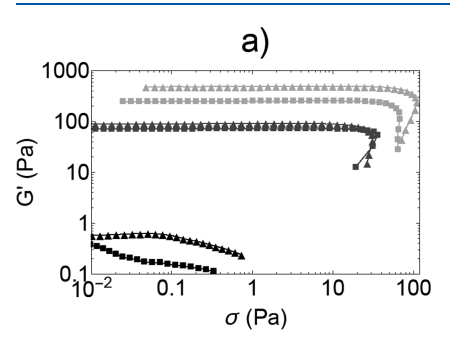

b)

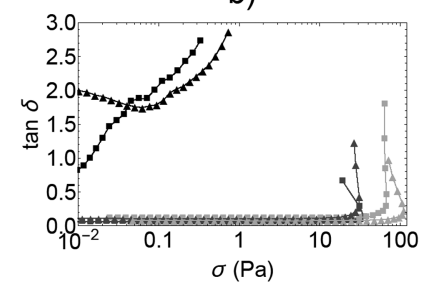

b)

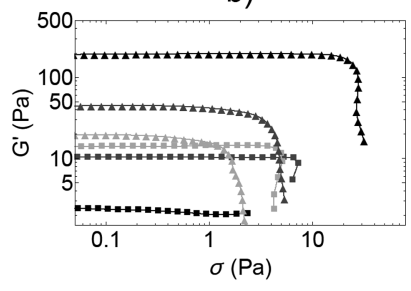

d)

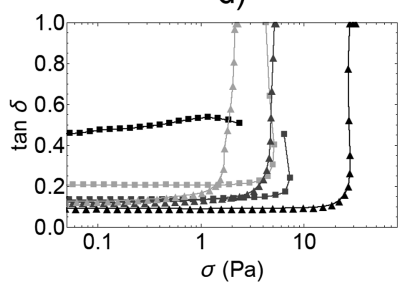

Figure 9. (a and b) Storage modulus $G^{\prime}$ and (c and d) $\tan \delta\left(=G^{\prime \prime} / G^{\prime}\right)$ versus stress curves for (a and c) OCNF and (b and d) CCNF dispersions and emulsions at $10 \mathrm{~g} / \mathrm{L}$ and various amounts of salt. Square symbols are associated with dispersions, and triangle symbols are associated with emulsions: (black) 0 , (dark gray) 0.1 , and (pale gray) $0.5 \mathrm{M} \mathrm{NaCl}$. Simultaneous plots of $G^{\prime}$ and $G^{\prime \prime}$ versus stress can be found in Figure $\mathrm{S} 13$.

carried out. For OCNF dispersions and emulsions without salt, the results show a fluid state. When salt is added, strong gel-like properties are found $\left(\tan \delta=G^{\prime \prime} / G^{\prime} \approx 0.1\right)$ with an increase of $G^{\prime}$ with increasing concentration of salt.

The gel properties $\left(G^{\prime}\right.$ and $\tan \delta$ ) are found to be similar for dispersions and emulsions. A similar behavior with the formation of a gel for CNC-stabilized emulsions in the presence of salt was previously observed. ${ }^{14}$ This proves that the gelation of emulsions can be triggered by the addition of salt, screening the electrostatic repulsion between droplets, in agreement with what has been observed for several other types of charged emulsions. ${ }^{12-14,16}$

For the CCNF-based systems, samples without salt evidence a higher $G^{\prime}$ and lower $\tan \delta$ for the emulsion than for the dispersion, once again consistent with the $\zeta$-potential measurements. Addition of salt results in an increase of $G^{\prime}$ for the dispersion and a decrease for the emulsion until it is found to be similar at $0.5 \mathrm{M}$, indicating that at this ratio the aggregation of the fibrils controls the rheological properties. Moreover, CCNF systems with salt show much weaker gel properties than their OCNF counterparts $\left(G^{\prime}=20\right.$ versus $400 \mathrm{~Pa}$ for emulsions at 0.5 $\mathrm{M} \mathrm{NaCl}$ and a yield stress almost 10 times lower) and are much less sensitive to salt, once again in agreement with the $\zeta$-potential measurements which show only a weak variation of the potential of the droplets with salt (from 24 to $13 \mathrm{mV}$ ). Notably, CCNF emulsions without salt have a $\zeta$-potential already below $+30 \mathrm{mV}$, often considered as a limiting value for dispersing colloids via electrostatic repulsion, explaining why a gel-like behavior is already observed for this system. When salt is added, it is suggested that, as for OCNF, the rheological properties are dominated by the behavior of the cellulose percolation network in the water phase, in agreement with previous studies. ${ }^{27,38}$ This explains why we find similar results for dispersions and emulsions.

In contrast to OCNF that bear negative charges thanks to the carboxyl groups that can be protonated, CCNF present fixed positive charges from the tetra-alkylammonium groups. ${ }^{43}$ This may explain the difference in the storage modulus between the two types of CNF for dispersions with the same amount of salt. For example, with a lower value of $|\zeta|$ for CCNF in water compared to OCNF, positively charged nanofibrils are less dispersible in water and more prone to uncontrolled aggregation with the addition of salt, resulting with a gel with a lower storage modulus.

Rheology results hence evidence a drastic change of the rheological properties of emulsions stabilized by OCNF, while CCNF-stabilized emulsions proved to be much less affected by the addition of salt. It is suggested that the rheological properties are due to the percolation network arising from the nanofibrils present in the thick shell stabilizing the oil droplets, in agreement with the CLSM, SANS, and $\zeta$-potential measurements.

\section{CONCLUSION}

Dispersed CNF bearing either negative or positive surface charges were used to form $\mathrm{o} / \mathrm{w}$ emulsions. In contrast to cellulose nanocrystals which form a well-defined Pickering monolayer at the oil-water interface, CNF-stabilized droplets present thick shells (typically above $100 \mathrm{~nm}$ ) with a hydration layer. In the case of OCNF, bearing negative charges, the influence of salt on the surface charge of the fibrils and subsequently the droplets allows tuning of the droplet aggregation state and hence alters the rheological properties of the sample, as observed using CLSM and rheology measurements. Indeed, tough gel-like properties were measured as $G^{\prime}$ increases with salt content alongside shear-thinning properties due to the breaking of the droplet clusters. CCNF, bearing positive charges, stabilize emulsions which are much less sensitive to salt, as evidenced by CLSM and rheology. This methodology allows tuning of the emulsion properties for particular applications, for example, entrapping oil droplets in gel-like material for formulation of creams and lotions in personal care.

\section{ASSOCIATED CONTENT}

\section{SI Supporting Information}

The Supporting Information is available free of charge at https://pubs.acs.org/doi/10.1021/acs.langmuir.0c03306.

Description of the onion model used to fit the SANS data; list of neutron scattering length densities (SLDs); SANS patterns of OCNF dispersions at various contrasts; OCNF contact angle at the hexadecane/water interface; droplet diameters of emulsions vs $\mathrm{pH}$; pictures of emulsions prior to/after centrifugation; CLSM micrographs of OCNF/CCNF emulsions with/without salt; 
SANS patterns of OCNF/CCNF emulsions with/without salt; tables of results from the SANS fitting; rheological studies of emulsions (PDF)

\section{AUTHOR INFORMATION}

\section{Corresponding Authors}

Karen J. Edler - Department of Chemistry, University of Bath, Bath BA2 7AY, United Kingdom; 이이.org/0000-00015822-0127; Email: K.Edler@bath.ac.uk

Janet L. Scott - Department of Chemistry and Centre for Sustainable Chemical Technologies, University of Bath, Bath BA2 7AY, United Kingdom; 10 orcid.org/0000-0001-80212860; Email: J.L.Scott@bath.ac.uk

\section{Authors}

James C. Courtenay - Department of Chemistry and Centre for Sustainable Chemical Technologies, University of Bath, Bath BA2 7AY, United Kingdom; $\odot$ orcid.org/0000-0003-12770697

Yun Jin - Department of Chemistry, University of Bath, Bath BA2 7AY, United Kingdom; ㅇo이.org/0000-0002-57246250

Julien Schmitt - Department of Chemistry, University of Bath, Bath BA2 7AY, United Kingdom; LSFC, Laboratoire de Synthèse et Fonctionnalisation des Céramiques, UMR 3080 CNRS/Saint-Gobain CREE, Saint-Gobain Research Provence, Cavaillon 84300, France; 이이. orcid.org/0000-0002-34526655

Kazi M. Zakir Hossain - Department of Chemistry, University of Bath, Bath BA2 7AY, United Kingdom

Najet Mahmoudi - ISIS Neutron \& Muon Source, STFC Rutherford Appleton Laboratory, Didcot OX11 OQX, United Kingdom

Complete contact information is available at:

https://pubs.acs.org/10.1021/acs.langmuir.0c03306

\section{Author Contributions}

\#J.C.C., Y.J., and J.S: These authors contributed equally.

\section{Notes}

The authors declare no competing financial interest.

SANS data supporting this paper are openly available from the ISIS Neutron and Muon Source Data Journal at https://data. isis.stfc.ac.uk/doi/INVESTIGATION/49913632/ and https:// data.isis.stfc.ac.uk/doi/STUDY/103200853/. All data supporting this paper are openly available from the University of Bath data archive at https://doi.org/10.15125/BATH-01013.

\section{ACKNOWLEDGMENTS}

The authors thank the EPSRC for funding this project (grant $\mathrm{EP} / \mathrm{N} 033310 / 1)$. We acknowledge ISIS for the provision of beamtime for the SANS experiments, and Dr. Ann Terry is thanked for her assistance with the first SANS experiment on Sans2d at the ISIS Neutron and Muon Source (experiment nos. RB1410091 and RB1710159). Dr. Ursula Potter is thanked for assistance in the acquisition of the TEM and SEM images and Anne Gesell for assistance in CLSM imaging. J.C.C. acknowledges funding from the EPSRC Centre for Doctoral Training in Sustainable Chemical Technology (EP/L016354/1).

\section{ABBREVIATIONS}

OCNF oxidized cellulose nanofibrils CCNF cationic cellulose nanofibrils
SLD scattering length density

\section{REFERENCES}

(1) Scott, J. L.; Lee, J. Appropriate lifetimes, fitting deaths. Green Chem. 2016, 18, 6157-6159.

(2) Klemm, D.; Heublein, B.; Fink, H.-P.; Bohn, A. Cellulose: Fascinating Biopolymer and Sustainable Raw Material. Angew. Chem., Int. Ed. 2005, 44, 3358-3393.

(3) Eichhorn, S. J. Cellulose nanowhiskers: promising materials for advanced applications. Soft Matter 2011, 7, 303-315.

(4) Saito, T.; Nishiyama, Y.; Putaux, J.-L.; Vignon, M.; Isogai, A. Homogeneous Suspensions of Individualized Microfibrils from TEMPO-Catalyzed Oxidation of Native Cellulose. Biomacromolecules 2006, 7 (6), 1687-1691.

(5) Isogai, A.; Saito, T.; Fukuzumi, H. TEMPO-oxidized cellulose nanofibers. Nanoscale 2011, 3, 71-85.

(6) Crawford, R. J.; Edler, K. J.; Lindhoud, S.; Scott, J. L.; Unali, G. Formation of shear thinning gels from partially oxidised cellulose nanofibrils. Green Chem. 2012, 14, 300-303.

(7) Crawford, R. J.; Scott, J. L.; Unali, G. F. Structured Aqueous detergents composition. PCT Patent Application, WO/2010/076292, 2010.

(8) Scott, J. L.; Smith, C.; Unali, G. F. Aqueous Gels. PCT Patent Application, WO/2012/171725, 2012.

(9) Smith, C.; Unali, G. F. Oil-in-water Emulsions. PCT Patent Application, WO/2012/139817, 2012.

(10) Pickering, S. U. Emulsions. J. Chem. Soc., Trans. 1907, 91, 20012021.

(11) Tcholakova, S.; Denkov, N. D.; Lips, A. Comparison of solid particles, globular proteins and surfactants as emulsifiers. Phys. Chem. Chem. Phys. 2008, 10, 1608-1627.

(12) Mezzenga, R.; Schurtenberger, P.; Burbidge, A.; Michel, M. Understanding foods as soft materials. Nat. Mater. 2005, 4, 729-740.

(13) Du Le, H.; Loveday, S. M.; Singh, H.; Sarkar, A. Pickering emulsions stabilised by hydrophobically modified cellulose nanocrystals: Responsiveness to $\mathrm{pH}$ and ionic strength. Food Hydrocolloids 2020, 99, 105344.

(14) Varanasi, S.; Henzel, L.; Mendoza, L.; Prathapan, R.; Batchelor, W.; Tabor, R.; Garnier, G. Pickering Emulsions Electrostatically Stabilized by Cellulose Nanocrystals. Front. Chem. 2018, 6, 409.

(15) Cheng, L.-C.; Sherman, Z. M.; Swan, J. W.; Doyle, P. S. Colloidal Gelation through Thermally Triggered Surfactant Displacement. Langmuir 2019, 35 (29), 9464-9473.

(16) Cheng, L.-C.; Kuei Vehusheia, S. L.; Doyle, P. S. Tuning Material Properties of Nanoemulsion Gels by Sequentially Screening Electrostatic Repulsions and Then Thermally Inducing Droplet Bridging. Langmuir 2020, 36, 3346-3355.

(17) Binks, B. P.; Lumsdon, S. O. Influence of Particle Wettability on the Type and Stability of Surfactant-Free Emulsions. Langmuir 2000, $16,8622-8631$

(18) Saleh, N.; Sarbu, T.; Sirk, K.; Lowry, G. V.; Matyjaszewski, K.; Tilton, R. D. Oil-in-Water Emulsions Stabilized by Highly Charged Polyelectrolyte-Grafted Silica Nanoparticles. Langmuir 2005, 21, 9873-9878.

(19) Kaptay, G. On the equation of the maximum capillary pressure induced by solid particles to stabilize emulsions and foams and on the emulsion stability diagrams. Colloids Surf., A 2006, 282-283, 387-401.

(20) Dugyala, V. R.; Daware, S. V.; Basavaraj, M. G. Shape anisotropic colloids: synthesis, packing behavior, evaporation driven assembly, and their application in emulsion stabilization. Soft Matter 2013, 9, 67116725.

(21) Kalashnikova, I.; Bizot, H.; Cathala, B.; Capron, I. New Pickering Emulsions Stabilized by Bacterial Cellulose Nanocrystals. Langmuir 2011, 27, 7471-7479.

(22) Kalashnikova, I.; Bizot, H.; Cathala, B.; Capron, I. Modulation of Cellulose Nanocrystals Amphiphilic Properties to Stabilize Oil/Water Interface. Biomacromolecules 2012, 13, 267-275. 
(23) Liu, L.; Hu, Z.; Sui, X.; Guo, J.; Cranston, E. D.; Mao, Z. Effect of Counterion Choice on the Stability of Cellulose Nanocrystal Pickering Emulsions. Ind. Eng. Chem. Res. 2018, 57, 7169-7180.

(24) Wang, W.; Du, G.; Li, C.; Zhang, H.; Long, Y.; Ni, Y. Preparation of cellulose nanocrystals from asparagus (Asparagusofficinalis L.) and their applications to palm oil/water Pickering emulsion. Carbohydr. Polym. 2016, 151, 1-8.

(25) Goi, Y.; Fujisawa, S.; Saito, T.; Yamane, K.; Kuroda, K.; Isogai, A. Dual Functions of TEMPO-Oxidized Cellulose Nanofibers in Oil-inWater Emulsions: A Pickering Emulsifier and a Unique Dispersion Stabilizer. Langmuir 2019, 35, 10920-10926.

(26) Aaen, R.; Brodin, F.; Simon, S.; Heggset, E.; Syverud, K. Oil-inWater Emulsions Stabilized by Cellulose Nanofibrils-The Effects of Ionic Strength and $\mathrm{pH}$. Nanomaterials 2019, 9, 259.

(27) Gestranius, M.; Stenius, P.; Kontturi, E.; Sjöblom, J.; Tammelin, T. Phase behaviour and droplet size of oil-in-water Pickering emulsions stabilised with plant-derived nanocellulosic materials. Colloids Surf., A 2017, 519, 60-70.

(28) Napso, S.; Rein, D. M.; Khalfin, R.; Kleinerman, O.; Cohen, Y. Cellulose gel dispersion: From pure hydrogel suspensions toencapsulated oil-in-water emulsions. Colloids Surf., B 2016, 137, 70-76.

(29) Alfassi, G.; Rein, D. M.; Cohen, Y. Cellulose emulsions and their hydrolysis. J. Chem. Technol. Biotechnol. 2019, 94 (1), 178-184.

(30) Araki, J. Electrostatic or steric? - preparations and characterizations of well-dispersed systems containing rod-like nanowhiskers of crystalline polysaccharides. Soft Matter 2013, 9, 4125-4141.

(31) Dong, X. M.; Revol, J.-F.; Gray, D. G. Effect of microcrystallite preparation conditions on the formation of colloid crystals of cellulose. Cellulose 1998, 5, 19-32.

(32) Kalashnikova, I.; Bizot, H.; Bertoncini, P.; Cathala, B.; Capron, I. Cellulosic nanorods of various aspect ratios for oil in water Pickering emulsions. Soft Matter 2013, 9, 952-959.

(33) Cherhal, F.; Cousin, F.; Capron, I. Structural Description of the Interface of Pickering Emulsions Stabilized by Cellulose Nanocrystals. Biomacromolecules 2016, 17, 496-502.

(34) Saidane, D.; Perrin, E.; Cherhal, F.; Guellec, F.; Capron, I. Some modification of cellulose nanocrystals for functional Pickering emulsions. Philos. Trans. R. Soc., A 2016, 374, 20150139.

(35) Zoppe, J. O.; Venditti, R. A.; Rojas, O. J. Pickering emulsions stabilized by cellulose nanocrystals grafted with thermo-responsive polymer brushes. J. Colloid Interface Sci. 2012, 369, 202-209.

(36) Tang, J.; Lee, M. F. X.; Zhang, W.; Zhao, B.; Berry, R. M.; Tam, K. C. Dual Responsive Pickering Emulsion Stabilized by Poly[2(dimethylamino)ethyl methacrylate] Grafted Cellulose Nanocrystals. Biomacromolecules 2014, 15, 3052-3060.

(37) Carrillo, C. A.; Nypelö, T. E.; Rojas, O. J. Cellulose nanofibrils for one-step stabilization of multiple emulsions (W/O/W) based on soybean oil. J. Colloid Interface Sci. 2015, 445, 166-173.

(38) Bai, L.; Huan, S.; Xiang, W.; Rojas, O. J. Pickering emulsions by combining cellulose nanofibrils and nanocrystals: phase behavior and depletion stabilization. Green Chem. 2018, 20, 1571-1582.

(39) Fujisawa, S.; Togawa, E.; Kuroda, K. Nanocellulose-stabilized Pickering emulsions and their applications. Sci. Technol. Adv. Mater. 2017, 18, 959-971.

(40) Jiménez-Saelices, C.; Capron, I. Design of Pickering micro and nanoemulsions based on the structural characteristics of nanocelluloses. Biomacromolecules 2018, 19, 460-469.

(41) Heise, K.; Kontturi, E.; Allahverdiyeva, Y.; Tammelin, T.; Linder, M. B.; Nonappa; Ikkala, O. Nanocellulose: Recent Fundamental Advances and Emerging Biological and Biomimicking Applications. Adv. Mater. 2021, 33, 2004349.

(42) Schmitt, J.; Calabrese, V.; Da Silva, M. A.; Lindhoud, S.; Alfredsson, V.; Scott, J. L.; Edler, K. J. TEMPO-oxidised cellulose nanofibrils; probing the mechanisms of gelation via Small Angle X-Ray Scattering. Phys. Chem. Chem. Phys. 2018, 20, 16012-16020.

(43) Courtenay, J. C.; Ramalhete, S. M.; Skuze, W. J.; Soni, R.; Khimyak, Y. Z.; Edler, K. J.; Scott, J. L. Unravelling cationic cellulose nanofibril hydrogel structure: NMR spectroscopy and small angle neutron scattering analyses. Soft Matter 2018, 14, 255-263.
(44) Jin, Y.; Edler, K. J.; Marken, F.; Scott, J. L. Voltammetric optimization of TEMPO-mediated oxidations at cellulose fabric. Green Chem. 2014, 16, 3322-3327.

(45) Zaman, M.; Xiao, H.; Chibante, F.; Ni, Y. Synthesis and characterization of cationically modified nanocrystalline cellulose. Carbohydr. Polym. 2012, 89, 163-170.

(46) Herth, W.; Schnepf, E. The Fluorochrome, Calcofluor White, Binds Oriented to Structural Polysaccharide Fibrils. Protoplasma 1980, 105, 129-133.

(47) Feigin, L. A.; Svergun, D. I. Structure Analysis by Small-Angle XRay and Neutron Scattering; Plenum Press: New York, 1987.

(48) Manet, S.; Lecchi, A.; Impéror-Clerc, M.; Zholobenko, V.; Durand, D.; Oliveira, C. L. P.; Pedersen, J. S.; Grillo, I.; Meneau, F.; Rochas, C. Structure of Micelles of a Nonionic Block Copolymer Determined by SANS and SAXS. J. Phys. Chem. B 2011, 115 (39), 11318-11329.

(49) Mao, Y.; Liu, K.; Zhan, C.; Geng, L.; Chu, B.; Hsiao, B. S. Characterization of Nanocellulose Using Small-Angle Neutron, X-ray, and Dynamic Light Scattering Techniques. J. Phys. Chem. B 2017, 121, $1340-1351$.

(50) Olszewska, A.; Eronen, P.; Johansson, L.-S.; Malho, J.-M.; Ankerfors, M.; Lindström, T.; Ruokolainen, J.; Laine, J.; Osterberg, M. The behaviour of cationic NanoFibrillar Cellulose in aqueous media. Cellulose 2011, 18, 1213-1226.

(51) Calabrese, V.; da Silva, M. A.; Schmitt, J.; Munoz-Garcia, J.; Gabrielli, V.; Scott, J. L.; Angulo, J.; Khimyak, Y. Z.; Edler, K. J. Surfactant controlled zwitterionic cellulose nanofibril dispersions. Soft Matter 2018, 14, 7793-7800.

(52) Perrin, E.; Bizot, H.; Cathala, B.; Capron, I. Chitin Nanocrystals for Pickering High Internal Phase Emulsions. Biomacromolecules 2014, $15,3766-3771$.

(53) Fukuzumi, H.; Tanaka, R.; Saito, T.; Isogai, A. Dispersion stability and aggregation behavior of TEMPO-oxidized cellulose nanofibrils in water as a function of salt addition. Cellulose 2014, 21, 1553-1559. 